\title{
Go-sha-jinki-Gan Alleviates Inflammation in Neurological Disorders via p38-TNF Signaling in the Central Nervous System
}

\author{
Shiying Jiang ${ }^{1} \cdot$ Kousuke Baba $^{1} \cdot$ Tatsusada Okuno $^{1} \cdot$ Makoto Kinoshita $^{1} \cdot$ Chi-Jing Choong $^{1} \cdot$ Hideki Hayakawa $^{1}$. \\ Hiroshi Sakiyama ${ }^{1} \cdot$ Kensuke Ikenaka $^{1} \cdot$ Seiichi Nagano ${ }^{1} \cdot$ Tsutomu Sasaki $^{1}$ • Munehisa Shimamura ${ }^{1,2}$. \\ Yoshitaka Nagai ${ }^{1,3} \cdot$ Keisuke Hagihara $^{4} \cdot$ Hideki Mochizuki $^{1}$
}

Accepted: 7 October 2020 / Published online: 20 October 2020

(C) The American Society for Experimental NeuroTherapeutics, Inc. 2020

\begin{abstract}
Go-sha-jinki-Gan (GJG) is a traditional Japanese herbal medicine. In clinical practice, GJG is effective against neuropathic pain and hypersensitivity induced by chemotherapy or diabetes. In our previous study using a chronic constriction injury mouse model, we showed that GJG inhibited microglia activation by suppressing the expression of tumor necrosis factor- $\alpha$ (TNF- $\alpha)$ and p38 mitogen-activated protein kinase (p38 MAPK) in the peripheral nervous system. To investigate whether GJG can suppress inflammation in the central nervous system (CNS) in the context of neurological disorders, we examined the effect of GJG on the activation of resident glial cells and on p38-TNF signaling in two mouse models of neurological disorders: the experimental autoimmune encephalomyelitis (EAE) model of multiple sclerosis and the 1-methyl-4-phenyl-1,2,3,6-tetrahydropyridine (MPTP) model of Parkinson's disease. GJG administration relieved the severity of clinical EAE symptoms and MPTP-induced inflammation by decreasing the number of microglia and the production of TNF- $\alpha$ in the spinal cord of EAE mice and the substantia nigra of MPTP-treated mice. Accordingly, GJG suppressed the phosphorylation of p38 in glial cells of these two mouse models. We conclude that GJG attenuates inflammation of the CNS by suppressing glial cell activation, followed by a decrease in the production of TNF- $\alpha$ via $\mathrm{p} 38$-TNF signaling.
\end{abstract}

Key Words Go-sha-jinki-Gan · inflammation · multiple sclerosis $\cdot 1$-methyl-4-phenyl-1,2,3,6-tetrahydropyridine $\cdot$ tumornecrosis factor- $\alpha \cdot \mathrm{p} 38$

Shiying Jiang, Kousuke Baba contributed equally as first authors; Keisuke Hagihara, Hideki Mochizuki contributed equally as corresponding authors to this work.

Electronic supplementary material The online version of this article (https://doi.org/10.1007/s13311-020-00948-w) contains supplementary material, which is available to authorized users.

Keisuke Hagihara

hagihara.keisuke@gmail.com

Hideki Mochizuki

hmochizuki@neurol.med.osaka-u.ac

1 Department of Neurology, Graduate School of Medicine, Osaka University, Suita, Osaka 565-0871, Japan

2 Department of Health Development and Medicine, Graduate School of Medicine, Osaka University, Suita, Osaka 565-0871, Japan

3 Department of Neurotherapeutics, Graduate School of Medicine, Osaka University, Suita, Osaka 565-0871, Japan

4 Department of Advanced Hybrid Medicine, Graduate School of Medicine, Osaka University, Suita, Osaka 565-0871, Japan

\section{Introduction}

Traditional Oriental herbal medicine is increasingly used worldwide. Go-sha-jinki-Gan (GJG), a well-known Kampo formula, is a traditional Japanese herbal medicine. In clinical settings, GJG has been widely used for overactive bladder, nocturia, meralgia, and numbness in elderly patients for many years in Japan [1-3]. Several clinical studies have confirmed that GJG relieves chemotherapy-induced peripheral neuropathic pain [4] and reduces insulin resistance in type 2 diabetes with diabetic neuropathy [5-7]. Regarding the mechanism of action of GJG, experimental animal studies have revealed that it suppresses the functional alteration of transient receptor potential channels in chemotherapy-induced neuropathy $[8$, 9]. In studies of diabetes-related complications, GJG improved platelet aggregation and diabetic neuropathy via the nitric oxide pathway. This formula is also suggested to have vasodilating and antinociceptive effects $[6,10-12]$. Our 
previous study showed that GJG upregulated peroxisome proliferator-activated receptor-gamma coactivator- $1 \alpha$, followed by a decrease in tumor necrosis factor- $\alpha$ (TNF- $\alpha$ ) expression in the muscle of senescence-accelerated mice [1]. In the chronic constriction injury (CCI) mouse model of neuropathic pain, GJG suppressed microglia activation, accompanied by a decrease in TNF- $\alpha$ and phosphorylation of p38 mitogenactivated protein kinases (p38 MAPK) in the spinal cord of this peripheral nerve injury model. Together, these studies show that GJG exerts anti-inflammatory effects in various disease models, such as chemotherapy-induced neuropathy, diabetic neuropathy, sarcopenia, and CCI [1, 2, 13-15], highlighting its pharmacological effects and suggesting possible underlying molecular mechanisms.

Inflammation contributes to the pathogenesis of classic immune-mediated neurological diseases (multiple sclerosis (MS)), acute traumatic and cerebrovascular disorders, and neurodegenerative diseases (Parkinson's disease (PD) and Alzheimer's disease (AD)) [16]. MS is a typical progressive inflammatory demyelinating and neurodegenerative disease of the CNS, featuring recurrent episodes of focal demyelination symptoms [17-19]. Proinflammatory cytokines play important roles in inflammation, demyelination, and cell death in MS [17, 20,21]. PD is one of the most common neurodegenerative diseases, characterized with dopaminergic (DA) neuron loss in the substantia nigra (SN) and motor/nonmotor dysfunction [22]. Several studies in a PD mouse model also showed the involvement of inflammation in the pathogenesis of PD [22-25]. To verify whether GJG protects against CNS inflammation in neurological disorders, we used the experimental autoimmune encephalomyelitis (EAE) animal model of MS and the 1-methyl-4-phenyl-1,2,3,6-tetrahydropyridine (MPTP)-induced model of PD.

TNF- $\alpha$ is crucial to disease progression in the EAE model and MPTP neurotoxicity towards DA neurons [26-29], and phosphorylation of $\mathrm{p} 38 \mathrm{MAPK}$, a regulatory factor of TNF- $\alpha$, is upregulated in both the EAE and MPTP mouse models [24, 30-32].To elucidate the mechanism of action of GJG, we focused on $\mathrm{p} 38$-driven TNF- $\alpha$ production in the spinal cord of EAE mice and the SN of MPTP mice. Considering the previously reported suppressive effect of GJG on TNF- $\alpha$, here, we demonstrate that GJG exerts an anti-inflammatory effect in the CNS by suppressing p38 phosphorylation and TNF- $\alpha$ production.

\section{Methods}

\section{Preparation of GJG Extracts}

Granules of powdered GJG extract were manufactured and provided by Tsumura \& Co. (Tokyo, Japan) according to Japanese and international manufacturing guidelines. The standard daily dose of GJG prescribed for human use is $7.5 \mathrm{~g}$, which contains $4.5 \mathrm{~g}$ of spraydried mixed extract. The 10 components of GJG are as follows: Rehmanniae radix, Achyranthis radix, Corni fructus, Dioscoreae rhizoma, Plantaginis semen, Alismatis rhizoma, hoelen, moutan cortex, Cinnamomi cortex, and heat-processed aconite tuber powder [1, 2]. We used the same $4 \%(\mathrm{w} / \mathrm{w})$ GJG-containing diet manufactured by Oriental Yeast Co., Ltd. (Tokyo, Japan) as reported in our previous paper $[1,2]$.

\section{EAE Animal Model}

Wild-type female C57BL/6J mice aged 8 weeks were purchased from SLC Japan (Hamamatsu, Japan) and maintained in a specific pathogen-free environment, where they were allowed to acclimate for 2 weeks before experiments. All mice were given free access to water and food. The experimental procedures were approved by the Animal Care and Use Committee of Osaka University Graduate School of Medicine. Mice were randomly divided into control (CT), EAE, EAE + GJG pretreatment, and $\mathrm{EAE}+\mathrm{GJG}$ posttreatment groups. In the $\mathrm{EAE}+\mathrm{GJG}$ pretreatment group, mice were given free access to a diet containing 4\% (w/w) GJG beginning 2 weeks before EAE induction and remained on this diet for the duration of the experiment. Mice in the EAE + GJG posttreatment group were given $4 \%(\mathrm{w} / \mathrm{w})$ GJG from the day of EAE onset (8th day after induction) and continued receiving this treatment until sacrifice. The $\mathrm{CT}$ and EAE groups were given a normal diet (Oriental Yeast Co., Ltd.) throughout the experiment.

\section{MPTP Animal Model of PD}

Wild-type male C57BL/6J mice aged 8 weeks were purchased from SLC Japan and maintained in a specific pathogen-free environment. Mice were randomly divided into CT, MPTP, and MPTP + GJG groups. In the MPTP + GJG group, mice were given free access to a diet containing $4 \%(\mathrm{w} / \mathrm{w}) \mathrm{GJG}$ beginning 1 month before MPTP administration and remained on this diet until sacrifice. The other conditions were the same as mentioned above in the "EAE Animal Model" section.

\section{Induction and Clinical Evaluation of EAE}

We used a modified version of our previously reported method [33, 34]. Briefly, female C57BL/6J mice aged 10 weeks were subcutaneously injected with $100 \mu \mathrm{g}$ myelin oligodendrocyte glycoprotein $(\mathrm{MOG})_{35-55}$ peptide (MEVGWYRSPFSRVVHLYRNGK, Bio-Synthesis, Inc., Lewisville, TX, USA) emulsified in complete Freund's adjuvant (CFA, BD Biosciences, San Diego, CA, USA) 
Fig. 1 GJG pretreatment ameliorated clinical

demyelination symptoms of EAE as assessed by clinical scores. (a) Clinical EAE disease score (EAE score) in the EAE group ( $n=15)$ and the EAE + GJG pre group (pretreated with GJG for 2 weeks and then treated for the remainder of the experiment; $n=15$ ). The symptoms of mice in the EAE + GJG pre group were mitigated.

Data represent the mean \pm s.e.m. $* p=0.0496$ by two-way

ANOVA. (b) Incidence, onset day, maximum score, and cumulative disease score of the EAE group and the EAE + GJG pre group. Data represent the mean \pm s.e.m. The cumulative score was calculated as the sum of the daily means a

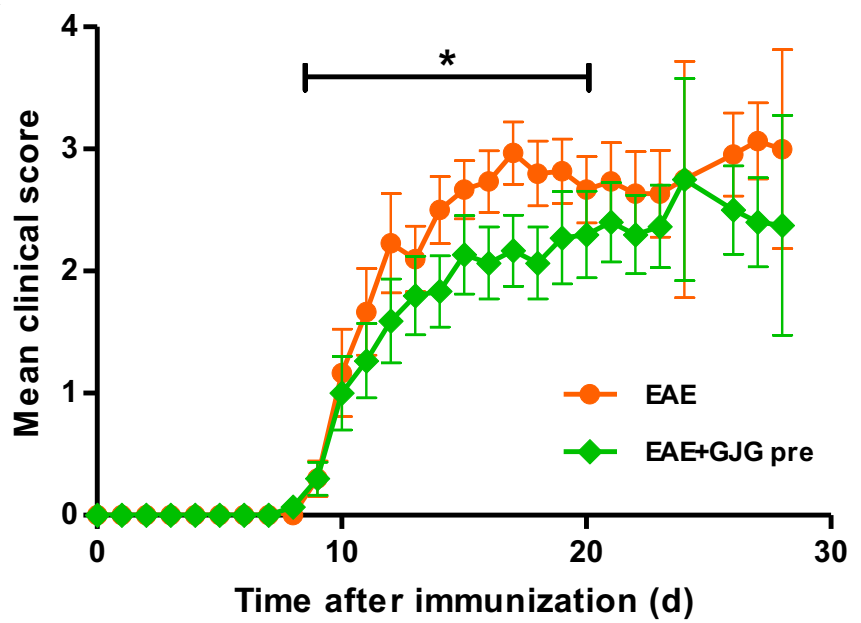

b

\begin{tabular}{|l|c|c|c|c|}
\hline & Incidence & Onset day & Maximum score & $\begin{array}{c}\text { Cumulative } \\
\text { disease score }\end{array}$ \\
\hline EAE & $100 \%(15 / 15)$ & $10.80 \pm 0.44$ & $3.13 \pm 0.36$ & $46.38 \pm 0.15$ \\
\hline EAE+GJG pre & $100 \%(15 / 15)$ & $11.33 \pm 0.90$ & $2.75 \pm 0.37$ & $37.96 \pm 0.15$ \\
\hline
\end{tabular}

containing $400 \mu \mathrm{g}$ Mycobacterium tuberculosis H37Ra (DIFCO Laboratories, Franklin Lakes, NJ, USA) following intraperitoneal injections of $200 \mathrm{ng}$ of pertussis toxin (List Biological Laboratories, Campbell, CA, USA) on days 0 and 2 . In the $\mathrm{EAE}+\mathrm{GJG}$ pretreatment group, mice were given a diet that contained $4 \%(\mathrm{w} / \mathrm{w})$ GJG beginning 2 weeks before immunization, whereas mice in the EAE + GJG posttreatment group were given $4 \%$ (w/w) GJG beginning on the predicted day of onset (the 8th day after EAE induction). All mice were monitored for clinical signs and scored on the following 0 to 5 scale until the day of sacrifice: 0 , no overt signs of disease; 0.5 , partially limp tail; 1, paralyzed tail; 1.5, abnormal righting reflex; 2 , gait abnormality; 2.5 , hind limb paresis; 3 , complete hind limb paralysis; 3.5, complete hind limb paralysis with enervation; 4, hind and front leg paralysis; 4.5, moribund state; 5, death by EAE [17]. Twenty-eight days after immunization, mice were sacrificed by transcardial perfusion with $4 \%$ paraformaldehyde (PFA) in PBS, and their spinal cords perfused with PBS were obtained for Western blotting analysis.

\section{MPTP Administration}

A modified version of an acute MPTP administration protocol was used. MPTP (Sigma-Aldrich, St. Louis, MO, USA) was dissolved in saline, and $15 \mathrm{mg} / \mathrm{kg}$ body weight was injected intraperitoneally into mice 4 times at 2-h intervals [35]. Seven days after injection, the mice were perfused with PBS, and SN samples were obtained; 4\% PFA perfusion was performed for immunohistochemical analysis.

\section{MOG-Specific T Cell Response and Cytokine Assay}

For the recall assay, spleens were collected from both GJG-treated and untreated EAE mice on the day of sacrifice. Splenocytes $\left(1 \times 10^{6}\right.$ cells $)$ were stimulated again with various concentrations of $\mathrm{MOG}_{35-55}$ peptide for $72 \mathrm{~h}$. Cytokines in the supernatant were assayed with mouse IFN- $\gamma$, IL-17, and IL-10 ELISA kits (R\&D Systems, Minneapolis, MN, USA) following the manufacturer's instructions.

\section{Histological and Immunohistochemical Analysis}

Paraffin-embedded blocks of lumbar spinal cords were cut into 4- $\mu \mathrm{m}$ sections for hematoxylin and eosin (H\&E) staining, Kluver-Barrera (KB) staining, and immunohistochemical staining. Histological quantification of H\&Estained sections was performed by counting the number of cells in an equal area in the same anatomical location in each group (3 sections per sample were selected for quantitative histological analysis). Frozen samples were cut into sections $10 \mu \mathrm{m}$ and $20 \mu \mathrm{m}$ thick. Sections obtained from paraffin-embedded blocks were dewaxed using Hemo-De (Falma Co., Ltd. Tokyo, Japan) and rehydrated in a descending ethanol series followed by distilled water. Antigen retrieval was performed by immersing the slides 
a

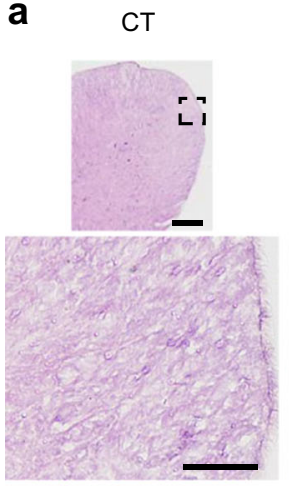

C

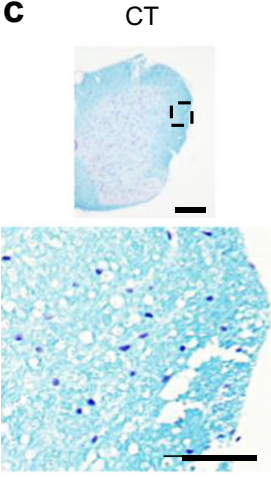

d

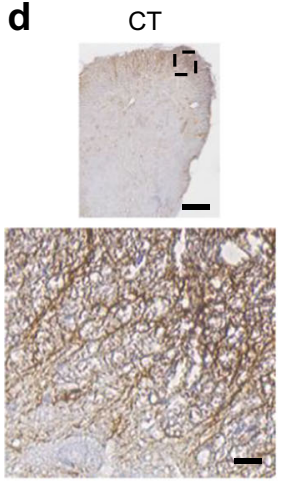

e

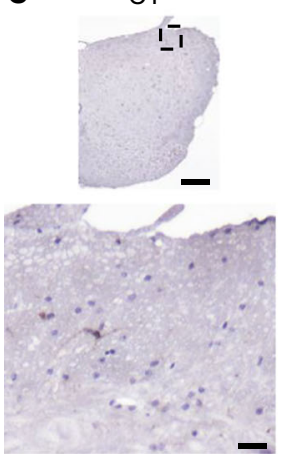

EAE

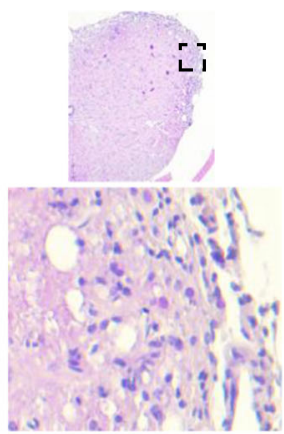

EAE
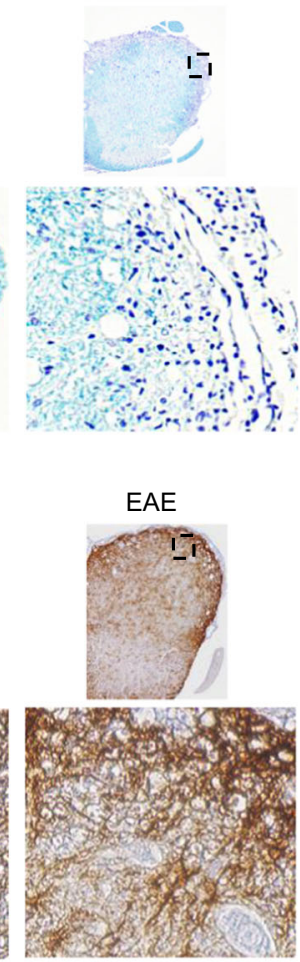

EAE

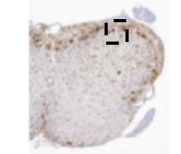

$E A E+G J G$ pre

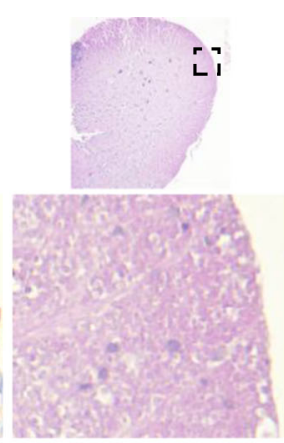

$E A E+G J G$ pre

b
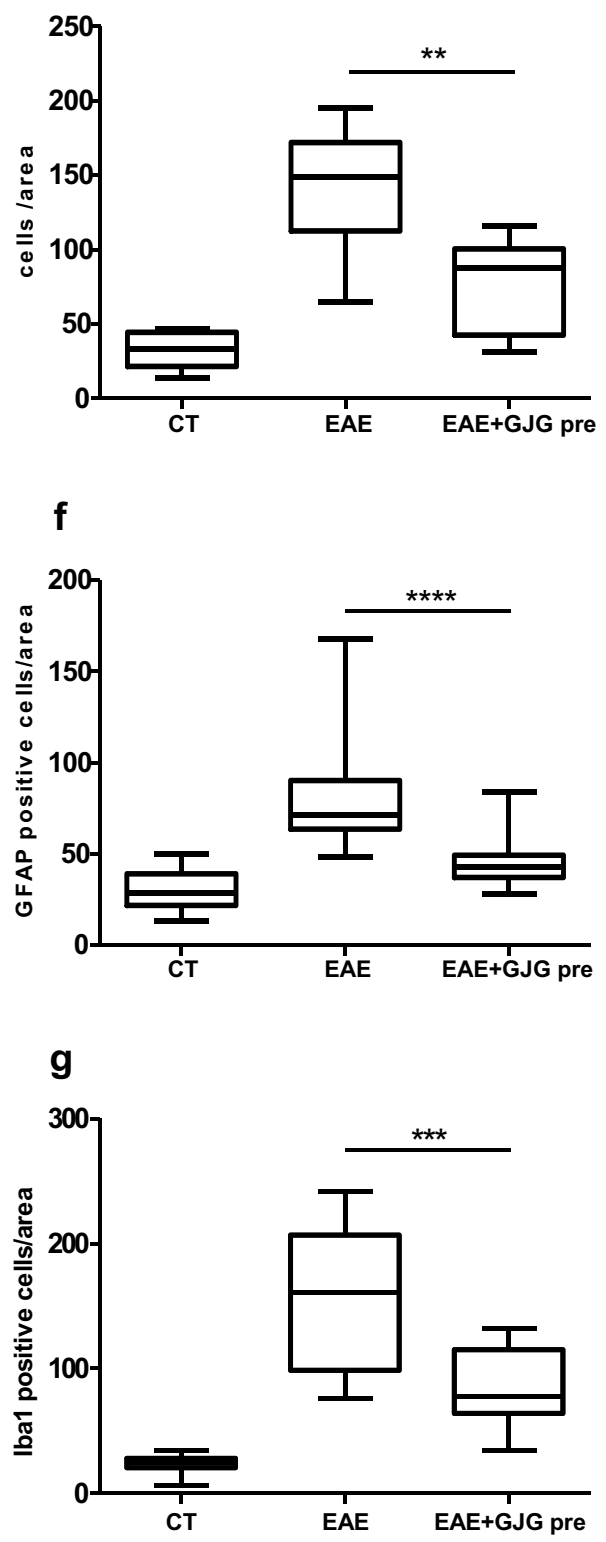

in $0.1 \mathrm{M}$ sodium citrate buffer ( $\mathrm{pH}$ 6) 3 times for $3 \mathrm{~min}$ each in a microwave oven or autoclaving them at $121{ }^{\circ} \mathrm{C}$ for $5 \mathrm{~min}$. After the endogenous peroxidase activity was quenched with $1 \% \mathrm{H}_{2} \mathrm{O}_{2}$ for $10 \mathrm{~min}$, the sections were washed with PBS 3 times for 5 min each and incubated overnight at $4{ }^{\circ} \mathrm{C}$ with the following primary antibodies: rabbit anti-tyrosine hydroxylase (TH, 1:5000, Calbiochem, Merck Millipore, Burlington, MA, USA), 
Fig. 2 GJG suppressed inflammatory cell infiltration, demyelination, and the numbers of GFAP-positive cells and Iba1-positive cells. (a) Hematoxylin and eosin (H\&E)-stained sections in the CT (mice without any treatment or immunization with $\mathrm{MOG}_{35-55}$ ) group, the EAE group, and the EAE + GJG pre group. (b) Histological quantification of H\&Estained sections in the CT group, the EAE group, and the EAE + GJG pre group ( $n=3$ per group). Cell infiltration in the lumbar spinal cord of the $\mathrm{EAE}+\mathrm{GJG}$ pre group decreased significantly. The horizontal bars inside the boxes represent the median values $(\mathrm{CT}=33.0, \mathrm{EAE}=149.0, \mathrm{EAE}+$ GJG pre $=88.0)$. $* * p=0.0051$, by the nonparametric Mann-Whitney $U$ test. (c) Kluver-Barrera (KB) staining of the lumbar spinal cord in the CT group, the EAE group, and the EAE + GJG pre group. The EAE + GJG pre group had a lesser degree of lumbar spinal cord demyelination than the EAE group. (d) GFAP immunohistochemical analysis of the lumbar spinal cord in the CT group, the EAE group, and the EAE + GJG pre group. (e) Ibal immunohistochemical analysis of the lumbar spinal cord in the CT group, the EAE group, and the EAE + GJG pre group. Scale bars: $a, b=200 \mu \mathrm{m}$ and $50 \mu \mathrm{m} ; \mathrm{c}, \mathrm{d}=200 \mu \mathrm{m}$ and $20 \mu \mathrm{m}$. (f) Box plot of the GFAP-positive cell counts of the CT group, the EAE group, and the $\mathrm{EAE}+\mathrm{GJG}$ pre group ( $n=3$ per group). In the EAE + GJG pre group, the number of GFAP-positive cells decreased significantly. The horizontal bars inside the boxes represent the median values $(\mathrm{CT}=28.5, \mathrm{EAE}=$ 71.5 , EAE + GJG pre $=42.5)$. $* * * * p<0.0001$ by the nonparametric Mann-Whitney $U$ test. (g) Box plot of the Iba1-positive cell counts of the CT group, EAE group, and EAE + GJG pre group ( $n=3$ per group). In the EAE + GJG pre group, the number of Iba1-positive cells decreased significantly. The horizontal bars inside the boxes represent the median values $(\mathrm{CT}=24.5, \mathrm{EAE}=161.0, \mathrm{EAE}+\mathrm{GJG}$ pre $=77.5) . * * * p=0.0001$ by the nonparametric Mann-Whitney $U$ test

rabbit anti-Iba1 (1:3000, Wako, Osaka, Japan), rabbit anti-glial fibrillary acidic protein (GFAP, 1:10000, Dako, Glostrup, Denmark), goat anti-Iba1 (1:500, Novus Biologicals, Centennial, CO, USA), rabbit antiTNF- $\alpha$ (1:25, Abcam, Cambridge, UK), rabbit anti-p38 (1:200, Cell Signaling Technology Inc. ((CST), Danvers, MA, USA), and rabbit anti-p-p38 (1:1600, CST). Sections were then washed with PBS 3 times for 5 min each and incubated for $1 \mathrm{~h}$ at room temperature with the following secondary antibodies EnVison+ System-HRP anti-rabbit IgG (1:1, Dako), FITC anti-goat IgG (1:500, Jackson ImmunoResearch, West Grove, PA, USA) and Cy3 antirabbit IgG (1:500, Jackson ImmunoResearch). For DAB coloration, sections were treated with the DAB Peroxidase Substrate Kit (Vector Laboratories, Burlingame, CA, USA) for $5 \mathrm{~min}$ and washed with PBS 3 times for 5 min each. Immunohistochemical and immunofluorescence images were obtained using an All-in-One Fluorescence Microscope BZ-X710 with BZ-X Analyzer Software (Keyence Corporation, Osaka, Japan) and an FV1200 Laser Scanning Microscope (BX61WI, Olympus Corporation, Tokyo, Japan) with the Fiji distribution of ImageJ (NIH, Bethesda, MD, USA). THpositive cell counts were performed using the unbiased stereology referring to our previous report [35]. Briefly, the number of TH- and Nissl-double positive neurons were counted every fourth section throughout the entire extent of the SN. The same location and the same area of each group (6 sections/sample) were defined as the quantitative area for counting GFAP- and Iba1-positive cells and Iba1- and TNF- $\alpha$-positive areas of dual immunofluorescence staining. For histological quantification of $\mathrm{H} \& \mathrm{E}-$ stained sections, the numbers of GFAP- and Iba1-positive cells were counted using BZ-X Analyzer Software (Keyence Corporation).

\section{Western Blotting Analysis}

The samples were homogenized in CelLytic MT Mammalian Tissue Lysis Reagent (Sigma-Aldrich) with a protease inhibitor cocktail (1:100, EMD Millipore Corp., Burlington, MA, USA) and a phosphatase inhibitor cocktail (1:100, Nacalai Tesque, Kyoto, Japan). Lysates were then centrifuged at $13500 \times \mathrm{g}$ for $20 \mathrm{~min}$ at $4{ }^{\circ} \mathrm{C}$, and protein levels were determined with a BCA Protein Assay Kit (Thermo Scientific, Waltham, MA, USA). Next, 10 to $20 \%$ gradient SDS-polyacrylamide gel electrophoresis (Wako) was performed, and the bands were transferred to PVDF membranes (Bio-Rad Laboratories, Hercules, CA, USA). After being blocked with 5\% skim milk for $1 \mathrm{~h}$ at room temperature, the membranes were incubated overnight at $4{ }^{\circ} \mathrm{C}$ with the following primary antibodies: goat anti-Iba1 (1:1000, Novus Biologicals), goat antiTNF- $\alpha$ (1:2000, R\&D Systems), rabbit anti-p38 (1:200, Santa Cruz Biotechnology, Dallas, TX, USA), rabbit antip-p38 (1:100, Santa Cruz Biotechnology) and mouse antiactin (1:10000, Merck Millipore). After being washed with $0.1 \%$ T-TBS buffer for $15-10-5 \mathrm{~min}$, the membranes were incubated with horseradish peroxidase (HRP)-conjugated secondary anti-goat antibody (1:10000, Santa Cruz Biotechnology), anti-mouse antibody (1:10000, GE Healthcare, Chicago, IL, USA) or anti-rabbit antibody (1:10000, GE Healthcare). Immunocomplexes were detected with a Chemi-Lumi One L Kit (Nacalai Tesque) and visualized with a ChemiDoc Touch Imaging System (Bio-Rad). Densitometry analysis was performed using Image Lab (Bio-Rad).

\section{Behavioral Assessment}

Mice were placed on a Rota-rod machine MK-630B (Muromachi Kikai Co., Ltd., Tokyo, Japan) under continuous acceleration from 4 to $40 \mathrm{rpm} / 300 \mathrm{~s}$ and the time mice stayed on the rod until falling/staying maximum for $5 \mathrm{~min}$ was recorded. Mice were trained 3 days before MPTP injection and Rota-rod assessment was performed on days $0,1,3,5$, and 7 after the injection. The reduction ratio is calculated by time on Rota-rod of the assessed day/the maximum time on Rota-rod of training day. 

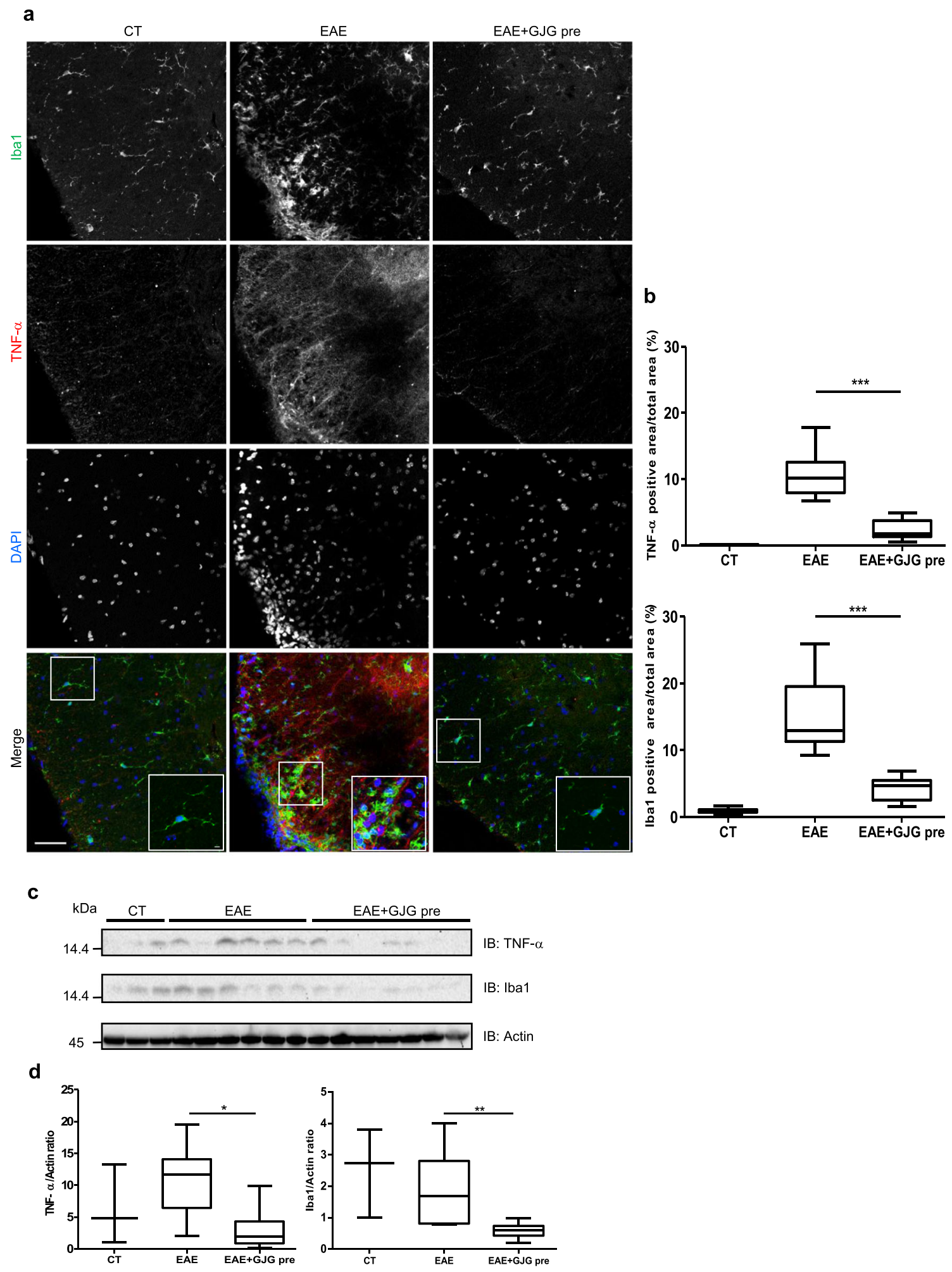

\section{Statistical Analysis}

Data are presented as the mean \pm s.e.m. for EAE clinical scores and cytokine assays. Statistical analysis of EAE clinical scores was performed by two-way
ANOVA. Histological quantification of H\&E-stained sections, GFAP-positive cell counts, Iba1-positive cell counts, TNF- $\alpha$-positive area, Iba1-positive area, THpositive cell counts, and quantification of Western blots were analyzed by the nonparametric Mann-Whitney $U$ 
Fig. 3 TNF- $\alpha$ and Ibal immunofluorescence and Western blotting analysis. (a) TNF- $\alpha$ and Iba1 dual staining immunofluorescence analysis of the lumbar spinal cord in the CT group, the EAE group, and the EAE + GJG pre group (red: TNF- $\alpha$, green: Iba1, blue: DAPI). TNF- $\alpha$ - and Iba1positive areas significantly decreased in the EAE + GJG pre group. Scale bars: $\mathrm{a}=50 \mu \mathrm{m}$ and $5 \mu \mathrm{m}$. (b) Box plot for quantification of TNF- $\alpha$ positive area and Iba1-positive area. The horizontal bars inside the boxes represent the median values $(\mathrm{TNF}-\alpha: \mathrm{CT}=0.1098, \mathrm{EAE}=10.18, \mathrm{EAE}+$ GJG pre $=1.768 ; \mathrm{Iba1}: \mathrm{CT}=0.9115, \mathrm{EAE}=12.90, \mathrm{EAE}+\mathrm{GJG}$ pre $=$ 4.667), $* * * p<0.0001$ in both TNF- $\alpha$ - and Iba1-positive areas, by the nonparametric Mann-Whitney $U$ test. (c) Western blotting analysis of TNF- $\alpha$ and Ibal obtained from the lumbar spinal cord in the CT group $(n=3)$, the EAE group $(n=6)$, and the EAE + GJG pre group $(n=7)$. A possible cleavage fragment of TNF- $\alpha(16 \mathrm{kDa})$ related to the soluble isoform 77-223 was detected. Actin was used as a loading control. (d) Box plot for quantification of TNF- $\alpha$ /actin and Iba1/actin ratios; both of these ratios decreased in the EAE + GJG pre group compared with the EAE group. The horizontal bars inside the boxes represent the median values $(\mathrm{TNF}-\alpha$ actin: $\mathrm{CT}=4.856, \mathrm{EAE}=11.69, \mathrm{EAE}+\mathrm{GJG}$ pre $=1.958$; Iba1/actin: $\mathrm{CT}=2.733, \mathrm{EAE}=1.679, \mathrm{EAE}+\mathrm{GJG}$ pre $=0.6105),{ }^{*} p=$ 0.0140 in TNF- $\alpha /$ actin, $* * p=0.0047$ in Iba1/actin, by the nonparametric Mann-Whitney $U$ test

test. Cytokine assays were analyzed by one-way ANOVA followed by Bonferroni's test. All data were analyzed by GraphPad Prism version 5 and version 6 (GraphPad Software, San Diego, CA, USA), the Statistical Package for Social Sciences version 23 (IBM, Armonk, NY, USA), and Excel (Microsoft, Redmond, WA, USA). Values of $p<0.05$ were considered significant.

\section{Results}

\section{GJG Reduced the Severity of Clinical Symptoms in EAE Mice}

To investigate whether GJG has an anti-inflammatory effect, we used the EAE mouse model. GJG pretreatment before immunization ameliorated disease severity at least slightly throughout the course of the disease, as assessed by the EAE clinical score. However, this treatment significantly attenuated clinical symptoms at the early stage of EAE after onset. (9th20 th day, $p=0.0496$; Fig. 1a). GJG seemed to have no influence on the disease incidence rate, onset day, maximum score, or cumulative score (Fig. 1b). As for GJG posttreatment, GJG mildly improved disease severity as measured by EAE clinical scores (Fig. S1a, b).

\section{GJG Suppressed Inflammatory Cell Infiltration, Reduced the Severity of Demyelination, and Suppressed Astrocytes and Microglia}

Because pretreatment with GJG attenuated disease severity as assessed by clinical scores, we suggested that the anti- inflammatory effect was one of the important factors acting against demyelination in EAE mice. Histological analysis and quantification of the mouse spinal cord showed that inflammatory cell infiltration and demyelination severity were significantly suppressed by GJG pretreatment and considerably suppressed by GJG posttreatment (Fig. 2a-c; in Fig. 2b, the median values are $\mathrm{CT}=33.0, \mathrm{EAE}=149.0, \mathrm{EAE}+\mathrm{GJG}$ post $=88.0, p=0.0051$; Fig. S2a $-\mathrm{c}$; in Fig. S2b, the median values are $\mathrm{CT}=36.0, \mathrm{EAE}=96.0, \mathrm{EAE}+\mathrm{GJG}$ post $=73.0$ ). Furthermore, markers of astrocytes (GFAP) and microglia (Iba1) in immunohistochemical analysis showed that GJG pretreatment suppressed the numbers of GFAP-positive cells and Iba1-positive cells (Fig. 2d, e). Quantification of the number of GFAP-positive cells and Iba1-positive cells in each group also illustrated that GJG pretreatment significantly suppressed the numbers of GFAP-positive cells and Iba1-positive cells (Fig. 2f, g; in Fig. 2f, the median values are $\mathrm{CT}=28.5$, $\mathrm{EAE}=71.5, \mathrm{EAE}+\mathrm{GJG}$ pre $=42.5, p<0.0001$; in Fig. $2 \mathrm{~g}$, the median values are $\mathrm{CT}=24.5, \mathrm{EAE}=161.0, \mathrm{EAE}+\mathrm{GJG}$ pre $=77.5, p=0.0001)$. In the EAE + GJG post group, there was a significant decrease in Iba1-positive cells; however, there was no significant change in the number of GFAPpositive cells (Fig. S2d-g; in Fig. S2f, the median values are $\mathrm{CT}=29, \mathrm{EAE}=76.5, \mathrm{EAE}+\mathrm{GJG}$ post $=69$; in Fig. $\mathrm{S} 2 \mathrm{~g}$, the median values are $\mathrm{CT}=23.5, \mathrm{EAE}=137.5, \mathrm{EAE}+\mathrm{GJG}$ post $=96, p=0.0018$ ). On this basis, we propose that GJG significantly ameliorates EAE by suppressing astrocytes and microglia.

\section{GJG Suppressed TNF-a Levels in EAE Mice}

Studies both in vitro and in vivo have suggested that TNF- $\alpha$ plays an important role in the pathology of MS [36]. We next analyzed TNF- $\alpha$ production by both immunohistochemical and biochemical analyses in the spinal cords of each group of mice. As a result, in dual immunofluorescence staining analysis of TNF- $\alpha$ and Iba1, we observed that both TNF- $\alpha$ and Iba1-positive areas were significantly suppressed in the $\mathrm{EAE}+\mathrm{GJG}$ pre group (Fig. 3a, b; in Fig. 3b, the median values of TNF- $\alpha$ are $\mathrm{CT}=0.1098, \mathrm{EAE}=10.18, \mathrm{EAE}+$ GJG pre $=1.768, p<0.0001$; the median values of Ibal are $\mathrm{CT}=0.9115, \mathrm{EAE}=12.90, \mathrm{EAE}+\mathrm{GJG}$ pre $=4.667, p<$ $0.0001)$. On the other hand, Western blotting analysis showed that both the level of TNF- $\alpha$ (specifically, a possible cleavage fragment of TNF- $\alpha(16 \mathrm{kDa})$ related to the soluble isoform 77-223) and the level of Ibal decreased significantly in the EAE + GJG pre group (Fig. 3c, d; in Fig. 3d, the median values of TNF- $\alpha$ actin are $\mathrm{CT}=4.856, \mathrm{EAE}=11.69, \mathrm{EAE}+$ GJG pre $=1.958, p=0.0140$; the median values of Iba1/actin are $\mathrm{CT}=2.733, \mathrm{EAE}=1.679, \mathrm{EAE}+\mathrm{GJG}$ pre $=0.6105, p=$ 0.0047). Pretreatment with GJG led to the suppression of TNF- $\alpha$ production and microglial counts. 

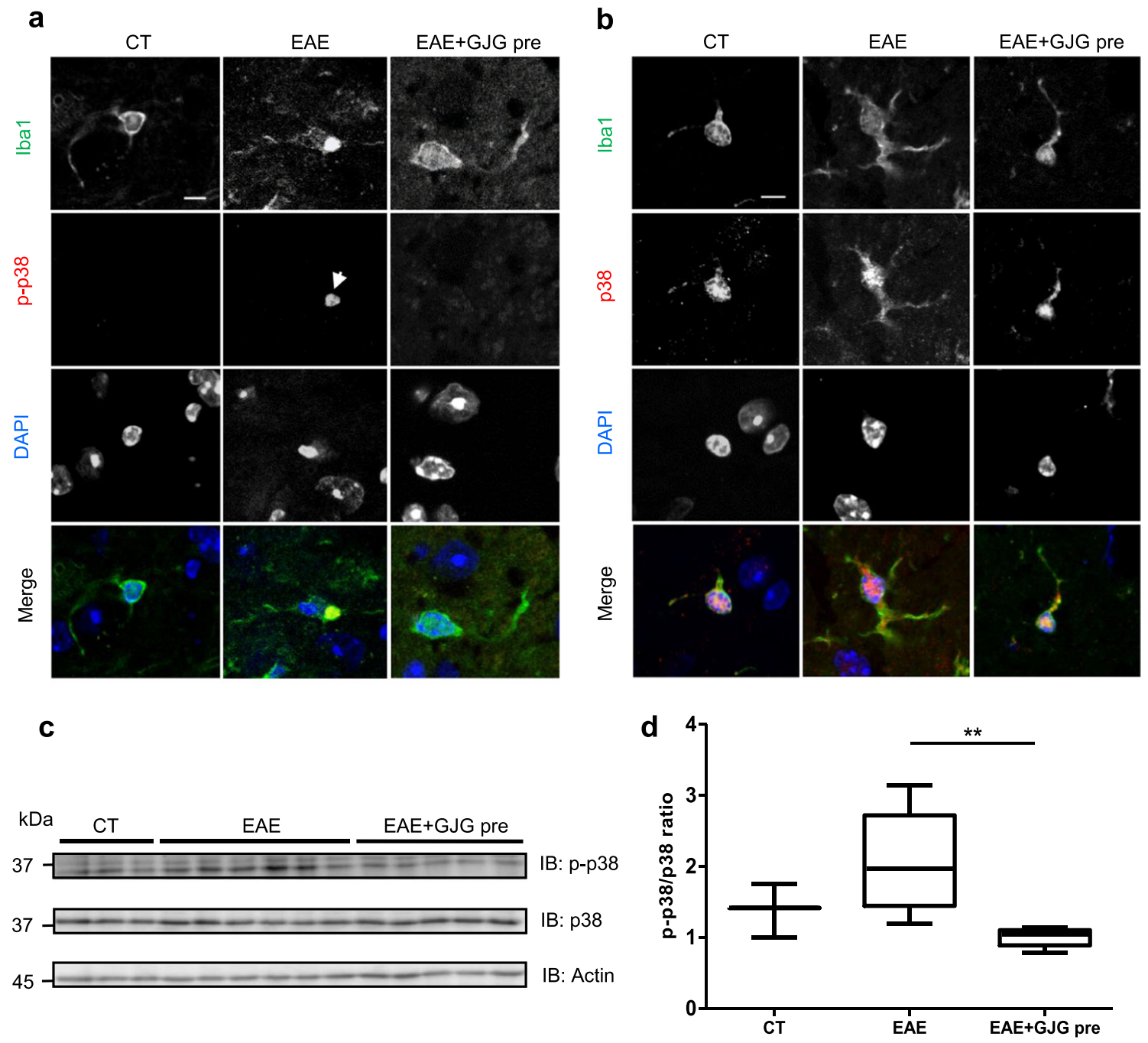

Fig. 4 p38 and p-p38 histochemical and Western blotting analysis of the CT group, the EAE group and the EAE + GJG pre group. (a) p-p38 positive signal on $\mathrm{p}-\mathrm{p} 38 / \mathrm{Iba}$ dual immunostaining was reduced (green: Iba1, red (arrow): p-p38, blue: DAPI). p-p38 was suppressed in the EAE + GJG pre group. Scale bars: $\mathrm{a}, \mathrm{b}=5 \mu \mathrm{m}$. (b) p38-positive signal in $\mathrm{p} 38 /$ Iba1 dual immunostaining showed no differences (green: Iba1, red: p-38, blue: DAPI). (c) Western blotting analysis of p38 and p-p38 in the CT

\section{GJG Suppressed P-38 MAPK Activation in the Spinal Cords of EAE Mice}

Because previous studies have reported that p38 is involved in the inflammation that occurs in EAE models, we examined whether GJG affects the phosphorylation of p38. In p38 and Iba1 dual staining of the spinal cord, phosphorylated p38 (p-p38), the signal pattern of which was consistent with a previous report [37], decreased in the EAE + GJG pre group and the EAE + GJG post group. However, total p38 expression was not significantly different among groups (Fig. 4a, b; Fig. S3a, b). Western blotting analysis of the EAE + GJG pre group also showed that the ratio of $\mathrm{p}-\mathrm{p} 38 / \mathrm{p} 38$ decreased significantly (Fig. 4c, d; in Fig. 4d, the median values are $\mathrm{CT}=$

group $(n=3)$, the EAE group $(n=6)$, and the EAE + GJG pre group $(n=$ 5). (d) Box plot for quantification of the p-p38/p38 ratio; this ratio decreased significantly in the EAE + GJG pre group compared with the EAE group. The horizontal bars inside the boxes represent the median values $(\mathrm{CT}=1.414, \mathrm{EAE}=1.964, \mathrm{EAE}+\mathrm{GJG}$ pre $=1.043), * * p=$ 0.0043 by the nonparametric Mann-Whitney $U$ test

$1.414, \mathrm{EAE}=1.964, \mathrm{EAE}+\mathrm{GJG}$ pre $=1.043, p=0.0043)$. This result indicated that GJG suppressed the phosphorylation of $\mathrm{p} 38$.

\section{GJG Had No Influence on the Priming Stage of Immunization}

During the lymphocyte proliferation priming stages of EAE before disease onset, proinflammatory cytokines such as interleukin (IL)-17, interferon (IFN)- $\gamma$, TNF- $\alpha$, and IL-10 play important roles in disease progression [20]. Suppressing those cytokines is thought to delay the onset. However, there were no apparent differences between GJG-treated and untreated EAE mice regarding the secretion level of any 
a

CT

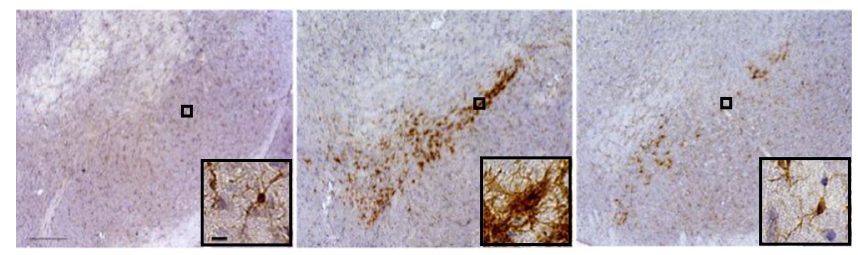

C

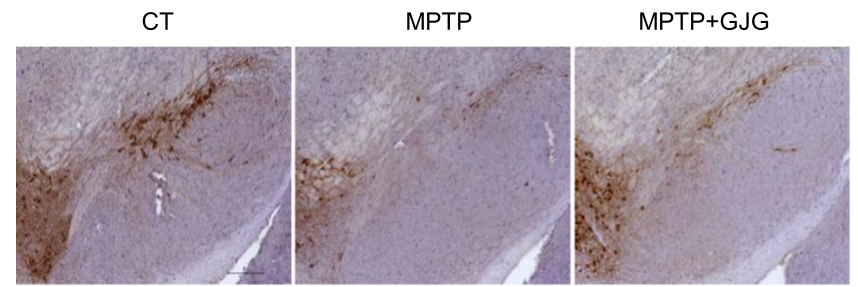

b

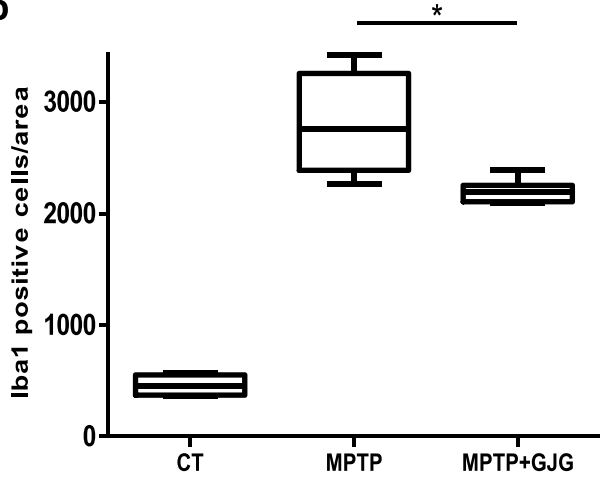

d

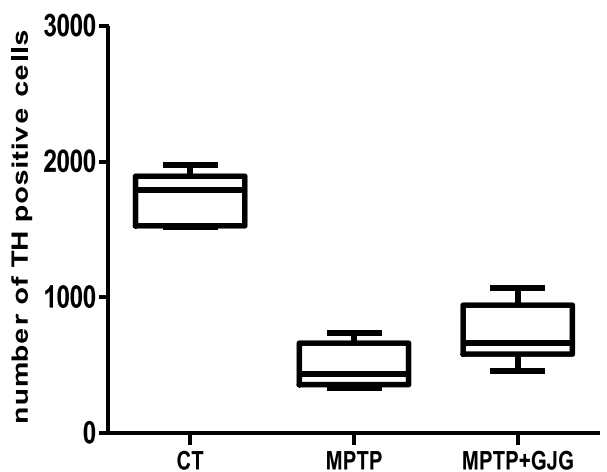

Fig. 5 Histological analysis of the CT group, the MPTP group, and the MPTP + GJG group. (a) Iba1-positive cell counts decreased in the MPTP group and the MPTP + GJG group. Scale bars: $\mathrm{a}=200 \mu \mathrm{m}$ and $10 \mu \mathrm{m}$. (b) Box plot for quantification of Iba1-positive cells. The number of Iba1positive cells decreased significantly in the MPTP + GJG group compared with the MPTP group. The horizontal bars inside the boxes represent the median values $(\mathrm{CT}=498, \mathrm{MPTP}=2870, \mathrm{MPTP}+\mathrm{GJG}=2156)$, * $p=0.019$ by the nonparametric Mann-Whitney $U$ test. (c)

proinflammatory cytokine (Fig. S4a-c). This result indicated that GJG has no influence on the onset of EAE.

\section{GJG Reduced the Number of Iba1-Positive Cells Without Attenuating DA Neuron Loss in MPTP- Injected Mice}

Histological analysis and quantification of Iba1-positive cells and TH (a marker of DA neurons)-positive cells in the SN showed that GJG suppressed the number of Iba1-positive cells compared with the number in the MPTP group (Fig. 5a, b; in Fig. $5 b$, the median values are $\mathrm{CT}=498, \mathrm{MPTP}=2870$, $\mathrm{MPTP}+\mathrm{GJG}=2156, p=0.019)$. Meanwhile, the number of TH-positive cells showed subtle change between the MPTP group and the MPTP + GJG group (Fig. 5c, d; in Fig. 5d, the median values are $\mathrm{CT}=1795, \mathrm{MPTP}=438.5, \mathrm{MPTP}+$ GJG $=665.5$ ), and Rota-rod behavioral test showed that GJG improved time on Rota-rod significantly in MPTP mice from 5 days after the last MPTP injection (Fig. S5, 5th day: $p=0.0256,7$ th day: $p=0.0161$ ). These results indicated that GJG had an anti-inflammatory effect in the CNS, which
Immunohistochemical analysis of brain tissue by TH staining in the CT group $(n=5)$, the MPTP group $(n=4)$, and the MPTP + GJG group $(n=$ 6). The numbers of TH-positive cells decreased in the MPTP group and the MPTP + GJG group. Scale bar: c $=200 \mu \mathrm{m}$. (d) Quantification of THpositive cells in the CT group, the MPTP group and the MPTP + GJG group. The horizontal bars inside the boxes represent the median values $(\mathrm{CT}=1795, \mathrm{MPTP}=438.5, \mathrm{MPTP}+\mathrm{GJG}=665.5)$

contributed to the recovery of motor deficit but this effect was not sufficient enough to improve the number of $\mathrm{TH}-$ positive cells significantly.

\section{GJG Suppressed TNF-a Production in the SN}

As mentioned above, GJG suppressed the production of TNF- $\alpha$ and p-p38 in the EAE model. We also investigated the level of TNF- $\alpha$ in the MPTP-induced PD model mice. In Western blotting analysis, the levels of both TNF- $\alpha$ production and Ibal significantly decreased in the MPTP + GJG group (Fig. 6; in Fig. 6b, the median values of TNF- $\alpha$ /actin are $\mathrm{CT}=1.100, \mathrm{MPTP}=2.052, \mathrm{MPTP}+\mathrm{GJG}=0.7867, p=$ 0.0159; the median values of Iba1/actin are CT $=1.199$, $\mathrm{MPTP}=1.628, \mathrm{MPTP}+\mathrm{GJG}=0.7467, p=0.0317)$.

\section{GJG Suppressed p38 MAPK Activation in MPTP- Injected Mice}

The expression of p-p38 and the ratio of p-p38 to p38 in the MPTP + GJG group also decreased significantly, whereas 
a

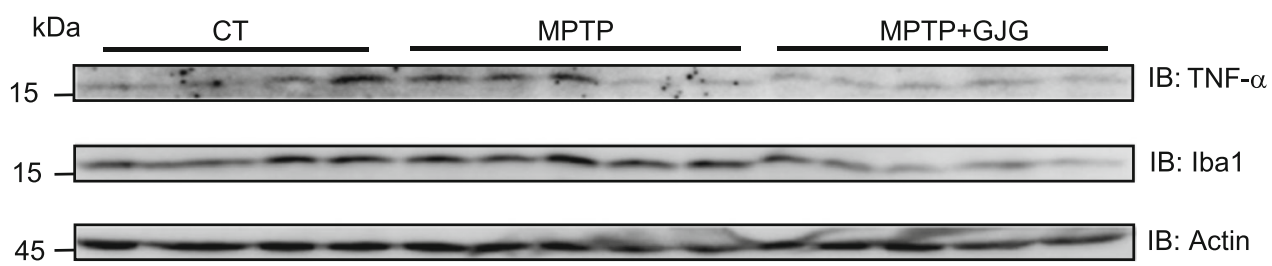

b

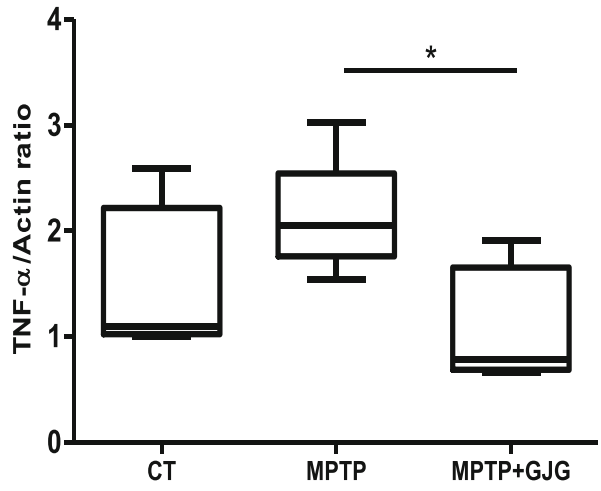

Fig. 6 TNF- $\alpha$ and Iba1 Western blotting analysis of the CT group, the MPTP group, and the MPTP + GJG group. (a) Western blotting analysis of TNF- $\alpha$ and Ibal obtained from SN in the CT group $(n=4)$, the MPTP group $(n=5)$, and the MPTP+GJG group $(n=5)$. A possible cleavage fragment of TNF- $\alpha(16 \mathrm{kDa})$ related to the soluble isoform 77-223 was detected. Actin was used as a loading control. (b) Box plot for quantification of the TNF- $\alpha$ /actin and Iba1/actin ratios; both ratios decreased in

total p-38 did not change (Fig. 7a, c, d; in Fig. 7d, the median values are $\mathrm{CT}=1.524, \mathrm{MPTP}=1.260, \mathrm{MPTP}+\mathrm{GJG}=$ $0.7604, p=0.0079)$. GJG also suppressed p-p38 in the $\mathrm{SN}$.

\section{Discussion}

In this study, we report that GJG treatment alleviates inflammation in the CNS degenerative disease models of MS and PD. Pathological analysis revealed that GJG suppressed resident glial cell activation, especially astrocytes and microglia. Decrease of TNF- $\alpha$ production accompanied by a reduction in p38 phosphorylation was also observed. Therefore, our results provide evidence that GJG protects against inflammation in the CNS.

It is well-known that inflammation plays an important role in the pathogenesis of both PNS and CNS diseases [38]. MS is a classic immune-mediated neurological condition in the $\mathrm{CNS}$, and the EAE mouse model is characterized by inflammation and demyelination of the spinal cord [16]. PD is a progressive neurodegenerative disease, and consistent findings obtained from various animal models of PD, including MPTP-based mouse model, suggest that inflammation

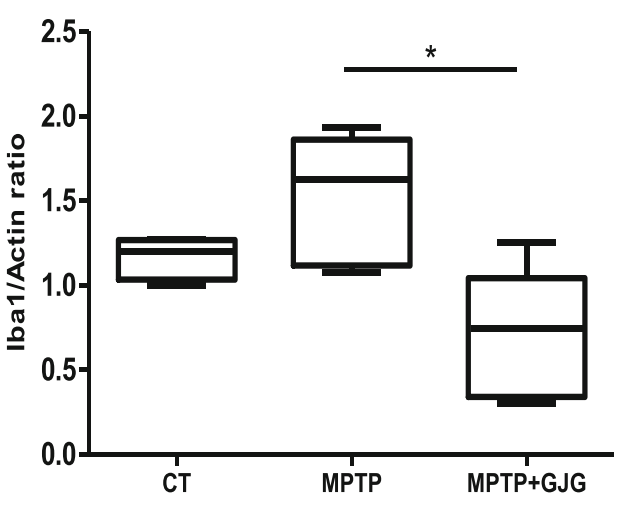

the MPTP + GJG group compared with the MPTP group. The horizontal bars inside the boxes represent the median values (TNF- $\alpha$ /actin: $\mathrm{CT}=$ $1.100, \mathrm{MPTP}=2.052, \mathrm{MPTP}+\mathrm{GJG}=0.7867 ; \mathrm{Iba} 1$ actin: $\mathrm{CT}=1.199$, $\mathrm{MPTP}=1.628, \mathrm{MPTP}+\mathrm{GJG}$ pre $=0.7467),{ }^{*} p=0.0159$ for TNF- $\alpha / \mathrm{ac}-$ tin, ${ }^{*} p=0.0317$ for Iba1/actin, by the nonparametric Mann-Whitney $U$ test

contributes to its pathogenesis and causes further damage to DA neurons [39]. Microglia are the resident immune cells of the CNS and relate to the pathogenesis of several neurological disorders. These cells are involved in promoting demyelination in MS [16, 40-46] and degeneration of DA neurons in PD [47]. High level of activated microglia were also found in the SN of PD patients [47, 48]. In our study, GJG alleviated the severity of symptoms by ameliorating inflammation and reducing the number of Iba1-positive cells in the spinal cord of EAE mice (Figs. 1 and 2) and MPTP mice (Fig. 5a, b). These results are consistent with the case of the CCI mouse model [2], suggesting the anti-inflammatory effect of GJG in the CNS.

In the EAE model, we found that GJG pretreatment was more effective than posttreatment (Figs. 1, 2, 3, and 4; Figs. S1-S3). Microglia can assume the M1 and M2 phenotypes, and macrophages/microglia involved in the different phases of EAE have different phenotypes [49, 50]. M1 phenotype polarization induced by TNF- $\alpha$ and IL- $1 \beta$ is usually associated with acute inflammation and the release of proinflammatory cytokines, whereas the M2 phenotype induced by IL- 4 and IL10 contributes to tissue repair $[49,51,52]$. In the Lewis rat EAE model, M1 macrophages contributed to disease 
a

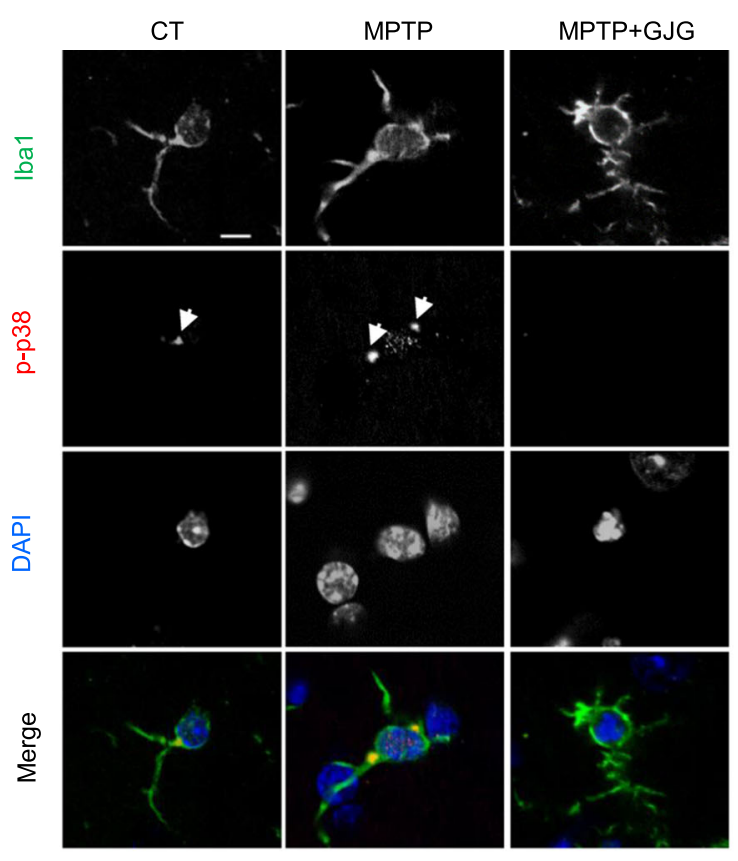

C

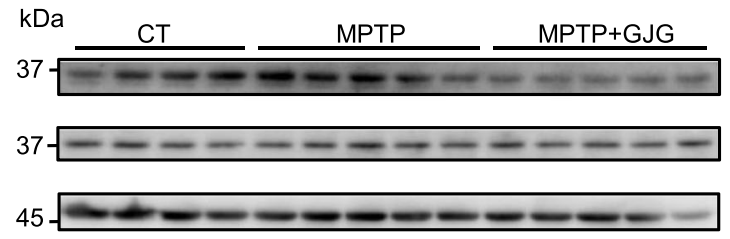

Fig. 7 p38 and p-p38 histochemical and Western blotting analysis of the CT group, the MPTP group, and the MPTP + GJG group. (a) p-p38 positive signal in p-p38/Ibal dual immunostaining was reduced (green: Iba1; red (arrow): p-p38; blue: DAPI). p-p38 was suppressed in the MPTP + GJG group. Scale bars $a, b=5 \mu \mathrm{m}$. (b) p38-positive signal in p38/Iba1 dual immunostaining showed no differences (green: Iba1; red: p-38; blue: DAPI). (c) Western blotting analysis of p38 and p-p38 in the

progression more than M2 macrophages at the early stage of EAE; on the other hand, M2 macrophages became predominant at the peak stage and the recovery stage [53]. Our findings suggest that GJG likely suppressed TNF- $\alpha$ secretion in M1 microglia and hence attenuated disease severity at the early stage.

By contrast, in the MPTP mouse model, there was only a subtle change but no significant differences were observed between the GJG-treated and untreated groups regarding the number of TH-positive neurons (Fig. 5c, d). On the other hand, Rota-rod behavioral test showed that although no significant differences were observed during the first 3 days, the motor deficit of MPTP + GJG mice significantly recovered b

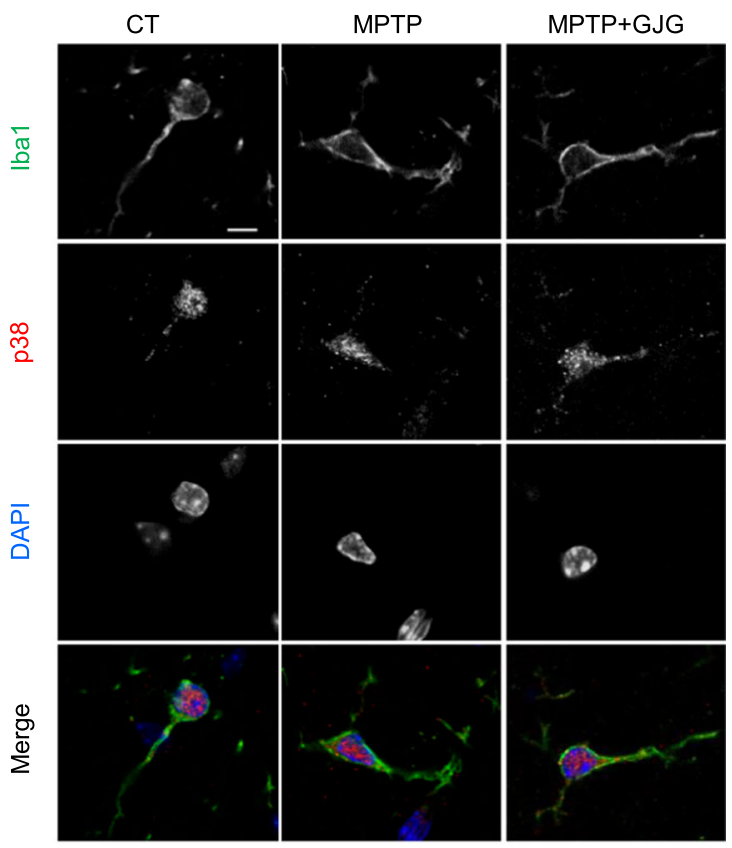

d

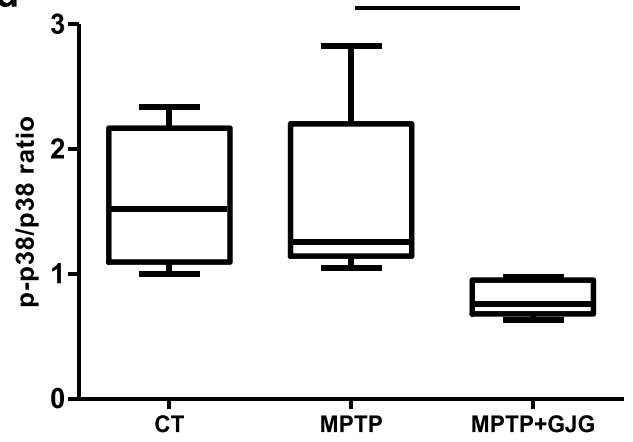

CT group ( $n=4)$, the MPTP group ( $n=5)$, and the MPTP + GJG group $(n=5)$. (d) Box plot for quantification of $\mathrm{p}-\mathrm{p} 38 / \mathrm{p} 38$. The $\mathrm{p}-\mathrm{p} 38 / \mathrm{p} 38$ ratio in the MPTP + GJG group decreased significantly compared with that in the MPTP group. The horizontal bars inside the boxes represent the median values $(\mathrm{CT}=1.524, \mathrm{MPTP}=1.260, \mathrm{MPTP}+\mathrm{GJG}=0.7604), * * p=$ 0.0079 , by the nonparametric Mann-Whitney $U$ test

after 5 days of MPTP injection (Fig. S5). GJG had positive influence on the recovery of motor deficit. In addition, the mRNA level of TNF- $\alpha$ also decreased in the striatum (ST) of MPTP + GJG mice (data not shown). The mechanism of action of MPTP involves multiple steps. Following systemic injection, MPTP rapidly crosses the blood-brain barrier and subsequently converted to the neurotoxic metabolite 1methyl-4-phenylpyridium (MPP+), which stimulates TNF- $\alpha$ production in glial cells. MPP+, a dopaminergic transporter substrate, can be taken up by DA neurons and concentrated in mitochondria, inhibiting mitochondrial complex I in the CNS and resulting in DA neuron loss [48]. The debris from dead neurons induces further activation of astrocytes and microglia. 
Cytokines from activated astrocytes and microglia then further damage DA neurons [39]. As mentioned above, mitochondria dysfunction and inflammation are two important factors of MPTP that cause DA neurons loss $[35,39,48]$. Considering these complex mechanisms, the motor deficit recovery of Rota-rod behavioral test, and the decline of TNF- $\alpha$ mRNA level in the ST of MPTP + GJG mice, we speculated that GJG significantly suppressed the inflammatory reaction in the midbrain, without affecting the toxicity of MPP+ to mitochondria. Suppressing inflammation alone promoted the recovery of motor deficit and subtly improved the number of THpositive cells; however, it did not influence DA neuron loss caused by mitochondria dysfunction. A better recovery effect is expectable given the anti-inflammatory effect of GJG. This is one possible explanation for why reduced inflammation in the SN was not parallel to DA neuron loss in GJG-treated mice.

TNF- $\alpha$ is one of the crucial proinflammatory cytokines in the induction and pathogenesis of several neurological disorders, including MS and PD [29, 54, 55]. Increased TNF- $\alpha$ stimulates glial cell activation and activated glial cells release more TNF- $\alpha$, forming a vicious cycle [26-29]. By reducing TNF- $\alpha$ production, GJG significantly prevented further EAE symptoms and inflammation induced by MPTP (Figs. 1, 2, 3, 5, and 6). Additionally, the TNF family is thought to contribute to the development of astrocyte proliferation in MS, and TNF- $\alpha$ reacts with astrocytes and foamy macrophages $[36,56]$. We observed that GJG pretreatment significantly suppressed GFAPpositive astrocytes (Fig. 2d, f). Based on this result, we propose that GJG also protected against inflammation by inhibiting the activation of astrocytes through the suppression of TNF- $\alpha$ production, as in the case of microglia.

Furthermore, p38 MAPK signaling plays an important role in the progression of EAE and can activate microglia and astrocytes in the CNS, regulating the production of TNF- $\alpha$ and related cytokines [57-62]. In the EAE rat model, p-p38 increased significantly [32]. A specific inhibitor of p38 (SB203580) suppressed the phosphorylation of p38 and significantly reduced the production of TNF- $\alpha$ in human microglia and astrocytes [63-65]. In the MPTP mouse model, the suppression of $\mathrm{p} 38$ activation decreased microglial activation and TNF- $\alpha$ release and mitigated further neuronal damage caused by the MPTP-induced inflammatory reaction [66]. The phosphorylation level of p38 was significantly suppressed in both the GJG-treated EAE and MPTP mouse models (Figs. 4 and 7). In contrast to p-p38, however, total p38 was unchanged. Therefore, we infer that GJG inhibited TNF- $\alpha$ production by suppressing $\mathrm{p} 38$ phosphorylation in the CNS. This is also consistent with our previous report examining the PNS [2].

Another possible pathway to ameliorate EAE symptoms is to suppress $\mathrm{T}$ cell priming and proliferation at the induction stage, delaying the onset of symptoms. In the EAE rodent model, IL-17, IL-10, and INF- $\gamma$ are important in the pathogenetic process [20]. As shown in Fig. S4, the production of related cytokines did not appear to differ between the EAE group and the EAE + GJG pre group, indicating that GJG did not affect $\mathrm{T}$ cell priming and proliferation at the early stage.

Although our previous study and the present study both showed that GJG has an anti-inflammatory effect, the mechanism of this effect is still not clear. The GJG formula contains 10 ingredients; one of these components, Bushi (aconite tuber, Fuzi) has an anti-inflammatory effect and inhibits astrocytic activation in neuropathic pain $[1,2,67,68]$. In rheumatoid arthritis animal models, Bushi also suppressed TNF- $\alpha$ production [67]. According to the studies described above, Bushi is likely the effective ingredient. However, even taking this into consideration, the exact active ingredient and mechanism of GJG remain uncertain.

Numerous evidence have shown that inflammatory process plays a key role in neurological diseases, such as MS, PD, AD, and amyotrophic lateral sclerosis [69-75]. Our study demonstrates that GJG can alleviate CNS inflammation and the involvement of $\mathrm{p} 38$-TNF signaling in its anti-inflammatory effect. Collectively, the main conclusion that can be drawn from our previous and current research finding is that GJG is effective against inflammation of neurological disorders. Because now GJG is already widely used and the consumption of natural herbal medicine is increasing rapidly in clinical settings $[1,2,76]$, our study opens up a new perspective on antiinflammatory effect of GJG in the CNS, and early use of GJG is suggested to gain a better effect to ameliorate the progression of inflammation-related neurological disorders.

Acknowledgments This research was supported by Grant-in-Aid for Scientific Research (C) Number JP17K09299 from Japan Society for the Promotion of Science (JSPS) and the Brain Mapping by Integrated Neurotechnologies for Disease Studies from Japan Agency for Medical Research and Development (AMED) Grant (19dm0207070).

Required Author Forms Disclosure forms provided by the authors are available with the online version of this article.

\section{References}

1. Kishida Y, Kagawa S, Arimitsu J, Nakanishi M, Sakashita N, Otsuka S, et al. Go-sha-jinki-Gan (GJG), a traditional Japanese herbal medicine, protects against sarcopenia in senescenceaccelerated mice. Phytomedicine. 2015;22(1):16-22.

2. Nakanishi M, Nakae A, Kishida Y, Baba K, Sakashita N, Shibata $\mathrm{M}$, et al. Go-sha-jinki-Gan (GJG) ameliorates allodynia in chronic constriction injury model mice via suppression of TNF-alpha expression in the spinal cord. Mol Pain 2016;12:1-16. 
3. Yagi H, Nishio K, Sato R, Arai G, Soh S, Okada H. Clinical efficacy and tolerability of Gosha-jinki-Gan, a Japanese traditional herbal medicine, for nocturia. J Tradit Complement Med 2016;6(1):126-9.

4. Cascella M, Muzio MR. Potential application of the Kampo medicine Goshajinkigan for prevention of chemotherapy-induced peripheral neuropathy. J Integr Med 2017;15(2):77-87.

5. Hirotani Y, Doi A, Ikeda K, Kato R, Ijiri Y, Tanaka K, et al. Effects of Gosha-jinki-Gan (Chinese herbal medicine: Niu-Che-Sen-QiWan) on hyperinsulinemia induced in rats fed a sucrose-rich diet. Drug Discov Ther 2011;5(4):181-4.

6. Hu X, Sato J, Oshida Y, Xu M, Bajotto G, Sato Y. Effect of Goshajinki-Gan (Chinese herbal medicine: Niu-Che-Sen-Qi-Wan) on insulin resistance in streptozotocin-induced diabetic rats. Diabetes Res Clin Pract 2003;59(2):103-11.

7. Uno T, Ohsawa I, Tokudome M, Sato Y. Effects of Goshajinkigan on insulin resistance in patients with type 2 diabetes. Diabetes Res Clin Pract 2005;69(2):129-35.

8. Mizuno K, Shibata K, Komatsu R, Omiya Y, Kase Y, Koizumi S. An effective therapeutic approach for oxaliplatin-induced peripheral neuropathy using a combination therapy with Goshajinkigan and Bushi. Cancer Biol Ther 2016;17(11):1206-12.

9. Mizuno K, Kono T, Suzuki Y, Miyagi C, Omiya Y, Miyano K, et al. Goshajinkigan, a traditional Japanese medicine, prevents oxaliplatin-induced acute peripheral neuropathy by suppressing functional alteration of TRP channels in rat. J Pharmacol Sci 2014;125(1):91-8.

10. Qin B, Nagasaki M, Ren M, Bajotto G, Oshida Y, Sato Y. Goshajinki-Gan (a herbal complex) corrects abnormal insulin signaling. Evid Based Complement Alternat Med 2004;1(3):269-76.

11. Suzuki Y, Goto K, Ishige A, Komatsu Y, Kamei J. Antinociceptive mechanism of Gosha-jinki-Gan in streptozotocin-induced diabetic animals: role of nitric oxide in the periphery. Jpn J Pharmacol 1999;79(3):387-91.

12. Suzuki Y, Goto K, Ishige A, Komatsu Y, Kamei J. Effect of Goshajinki-Gan, a Kampo medicine, on enhanced platelet aggregation in streptozotocin-induced diabetic rats. Jpn J Pharmacol 1998;78(1): 87-91.

13. Dalle S, Rossmeislova L, Koppo K. The role of inflammation in age-related sarcopenia. Front Physiol 2017;8:1045.

14. Areti A, Yerra VG, Naidu V, Kumar A. Oxidative stress and nerve damage: role in chemotherapy induced peripheral neuropathy. Redox Biol 2014;2:289-95.

15. Pop-Busui R, Ang L, Holmes C, Gallagher K, Feldman EL. Inflammation as a therapeutic target for diabetic neuropathies. Curr Diab Rep 2016;16(3):29.

16. Wee Yong V. Inflammation in neurological disorders: a help or a hindrance? Neuroscientist. 2010;16(4):408-20.

17. Stromnes IM, Goverman JM. Active induction of experimental allergic encephalomyelitis. Nat Protoc 2006;1(4):1810-9.

18. Escribano BM, Medina-Fernandez FJ, Aguilar-Luque M, Aguera E, Feijoo M, Garcia-Maceira FI, et al. Lipopolysaccharide binding protein and oxidative stress in a multiple sclerosis model. Neurotherapeutics. 2017;14(1):199-211.

19. Freedman SN, Shahi SK, Mangalam AK. The "gut feeling": breaking down the role of gut microbiome in multiple sclerosis. Neurotherapeutics. 2018;15(1):109-25.

20. Kaltsonoudis E, Voulgari PV, Konitsiotis S, Drosos AA. Demyelination and other neurological adverse events after antiTNF therapy. Autoimmun Rev 2014;13(1):54-8.

21. Sonar S, Lal G. Role of Tumor Necrosis Factor Superfamily in Neuroinflammation and Autoimmunity. Front Immunol. 2015;6: 364:1-13.

22. Glass CK, Saijo K, Winner B, Marchetto MC, Gage FH. Mechanisms underlying inflammation in neurodegeneration. Cell. 2010;140(6):918-34.
23. Saijo K, Winner B, Carson CT, Collier JG, Boyer L, Rosenfeld MG, et al. A Nurr1/CoREST pathway in microglia and astrocytes protects dopaminergic neurons from inflammation-induced death. Cell. 2009;137(1):47-59.

24. He J, Zhong W, Zhang M, Zhang R, Hu W. P38 mitogen-activated protein kinase and Parkinson's disease. Transl Neurosci 2018;9: 147-53.

25. Przedborski S, Vila M. MPTP: a review of its mechanisms of neurotoxicity. Clin Neurosci Res 2001;1(6):407-18.

26. Ruddle NH, Bergman CM, Mcgrath KM, Lingenheld EG, Grunnet ML, Padula SJ, et al. An antibody to lymphotoxin and tumornecrosis-factor prevents transfer of experimental allergic encephalomyelitis. J Exp Med 1990;172(4):1193-200.

27. Selmaj K, Raine CS, Cross AH. Anti-tumor necrosis factor therapy abrogates autoimmune demyelination. Ann Neurol 1991;30(5): 694-700.

28. Pachner AR. Experimental models of multiple sclerosis. Curr Opin Neurol 2011;24(3):291-9.

29. Sriram K, Matheson JM, Benkovic SA, Miller DB, Luster MI, O'Callaghan JP. Deficiency of TNF receptors suppresses microglial activation and alters the susceptibility of brain regions to MPTP-induced neurotoxicity: role of TNF-alpha. FASEB J 2006;20(6):670-82.

30. Coulthard LR, White DE, Jones DL, McDermott MF, Burchill SA. p38(MAPK): stress responses from molecular mechanisms to therapeutics. Trends Mol Med 2009;15(8):369-79.

31. Namiki K, Matsunaga H, Yoshioka K, Tanaka K, Murata K, Ishida $\mathrm{J}$, et al. Mechanism for p38alpha-mediated experimental autoimmune encephalomyelitis. J Biol Chem 2012;287(29):24228-38.

32. Shin T, Ahn M, Jung K, Heo S, Kim D, Jee Y, et al. Activation of mitogen-activated protein kinases in experimental autoimmune encephalomyelitis. J Neuroimmunol 2003;140(1-2):118-25.

33. Koda T, Okuno T, Takata K, Honorat JA, Kinoshita M, Tada S, et al. Sema4A inhibits the therapeutic effect of IFN-beta in EAE. J Neuroimmunol 2014;268(1-2):43-9.

34. Takata K, Kinoshita M, Okuno T, Moriya M, Kohda T, Honorat JA, et al. The lactic acid bacterium Pediococcus acidilactici suppresses autoimmune encephalomyelitis by inducing IL-10producing regulatory T cells. PLoS One 2011;6(11):e27644.

35. Furuya T, Hayakawa H, Yamada M, Yoshimi K, Hisahara S, Miura $\mathrm{M}$, et al. Caspase-11 mediates inflammatory dopaminergic cell death in the 1-methyl-4-phenyl-1,2,3,6-tetrahydropyridine mouse model of Parkinson's disease. J Neurosci 2004;24(8):1865-72.

36. Selmaj KW. Tumour necrosis factor and anti-tumour necrosis factor approach to inflammatory demyelinating diseases of the central nervous system. Ann Rheum Dis 2000;59 Suppl 1:194-102.

37. Kim JE, Park H, Choi SH, Kong MJ, Kang TC. Roscovitine Attenuates Microglia Activation and Monocyte Infiltration via p38 MAPK Inhibition in the Rat Frontoparietal Cortex Following Status Epilepticus. Cells. 2019;8(7):746:1-15.

38. Skaper SD, Facci L, Zusso M, Giusti P. An inflammation-centric view of neurological disease: beyond the neuron. Front Cell Neurosci 2018;12:72.

39. Episcopo FL, Tirolo C, Testa N, Caniglia S, Morale MC, Marchetti B. Reactive astrocytes are key players in nigrostriatal dopaminergic neurorepair in the MPTP mouse model of Parkinson's disease: focus on endogenous neurorestoration. Curr Aging Sci 2013;6(1):4555 .

40. Rawji KS, Yong VW. The benefits and detriments of macrophages/ microglia in models of multiple sclerosis. Clin Dev Immunol. 2013;2013:948976:1-13.

41. Kierdorf K, Erny D, Goldmann T, Sander V, Schulz C, Perdiguero EG, et al. Microglia emerge from erythromyeloid precursors via Pu.1- and Irf8-dependent pathways. Nat Neurosci 2013;16(3): 273-80. 
42. Li G, Yamasaki R, Fang M, Masaki K, Ochi H, Matsushita T, et al. Novel disease-modifying anti-rheumatic drug iguratimod suppresses chronic experimental autoimmune encephalomyelitis by down-regulating activation of macrophages/microglia through an NF-kappaB pathway. Sci Rep 2018;8(1):1933.

43. Kutzelnigg A, Lucchinetti CF, Stadelmann C, Bruck W, Rauschka $\mathrm{H}$, Bergmann $\mathrm{M}$, et al. Cortical demyelination and diffuse white matter injury in multiple sclerosis. Brain. 2005;128(Pt 11):270512.

44. Farias AS, de la Hoz C, Castro FR, Oliveira EC, Ribeiro dos Reis JR, Silva JS, et al. Nitric oxide and TNFalpha effects in experimental autoimmune encephalomyelitis demyelination. Neuroimmunomodulation. 2007;14(1):32-8.

45. Crocker SJ, Whitmire JK, Frausto RF, Chertboonmuang P, Soloway PD, Whitton JL, et al. Persistent macrophage/microglial activation and myelin disruption after experimental autoimmune encephalomyelitis in tissue inhibitor of metalloproteinase-1deficient mice. Am J Pathol 2006;169(6):2104-16.

46. Rasmussen S, Wang Y, Kivisakk P, Bronson RT, Meyer M, Imitola $\mathrm{J}$, et al. Persistent activation of microglia is associated with neuronal dysfunction of callosal projecting pathways and multiple sclerosislike lesions in relapsing - remitting experimental autoimmune encephalomyelitis. Brain. 2007;130:2816-29.

47. Block ML, Zecca L, Hong JS. Microglia-mediated neurotoxicity: uncovering the molecular mechanisms. Nat Rev Neurosci 2007;8(1):57-69.

48. Wichmann T, DeLong MR. MPTP parkinsonism model. In: Encyclopedia of Neuroscience 2009;1077-1081 [online]. Available at: https://doi.org/10.1016/B978-008045046-9.01295-X.

49. Chu F, Shi M, Zheng C, Shen D, Zhu J, Zheng X, et al. The roles of macrophages and microglia in multiple sclerosis and experimental autoimmune encephalomyelitis. J Neuroimmunol 2018;318:1-7.

50. Jiang Z, Jiang JX, Zhang GX. Macrophages: a double-edged sword in experimental autoimmune encephalomyelitis. Immunol Lett 2014;160(1):17-22.

51. Goldmann T, Prinz M. Role of microglia in CNS autoimmunity. Clin Dev Immunol 2013;2013:208093.

52. Aguilera G, Colin-Gonzalez AL, Rangel-Lopez E, Chavarria A, Santamaria A. Redox signaling, neuroinflammation, and neurodegeneration. Antioxid Redox Signal 2018;28(18):1626-51.

53. Shin T, Ahn M, Matsumoto Y. Mechanism of experimental autoimmune encephalomyelitis in Lewis rats: recent insights from macrophages. Anat Cell Biol 2012;45(3):141-8.

54. Sharief MK, Hentges R. Association between tumor-necrosisfactor-alpha and disease progression in patients with multiple-sclerosis. New Engl J Med 1991;325(7):467-72.

55. Gao H, Danzi MC, Choi CS, Taherian M, Dalby-Hansen C, Ellman DG, et al. Opposing functions of microglial and macrophagic TNFR2 in the pathogenesis of experimental autoimmune encephalomyelitis. Cell Rep 2017;18(1):198-212.

56. Selmaj KW, Farooq M, Norton WT, Raine CS, Brosnan CF. Proliferation of astrocytes in vitro in response to cytokines. A primary role for tumor necrosis factor. J Immunol 1990;144(1):12935.

57. Krementsov DN, Thornton TM, Teuscher C, Rincon M. The emerging role of p38 mitogen-activated protein kinase in multiple sclerosis and its models. Mol Cell Biol 2013;33(19):3728-34.

58. Boyle DL, Jones TL, Hammaker D, Svensson CI, Rosengren S, Albani S, et al. Regulation of peripheral inflammation by spinal p38 MAP kinase in rats. PLoS Med 2006;3(9):e338.

59. Kaminska B, Gozdz A, Zawadzka M, Ellert-Miklaszewska A, Lipko M. MAPK signal transduction underlying brain inflammation and gliosis as therapeutic target. Anat Rec (Hoboken) 2009;292(12):1902-13.

60. Han J, Brown T, Beutler B. Endotoxin-responsive sequences control cachectin/tumor necrosis factor biosynthesis at the translational level. J Exp Med 1990;171(2):465-75.

61. Kontoyiannis D, Pasparakis M, Pizarro TT, Cominelli F, Kollias G. Impaired on/off regulation of TNF biosynthesis in mice lacking TNF AU-rich elements: implications for joint and gut-associated immunopathologies. Immunity. 1999;10(3):387-98.

62. Dumitru CD, Ceci JD, Tsatsanis C, Kontoyiannis D, Stamatakis K, Lin $\mathrm{JH}$, et al. TNF-alpha induction by LPS is regulated posttranscriptionally via a Tpl2/ERK-dependent pathway. Cell. 2000;103(7):1071--83.

63. ten Hove T, van den Blink B, Pronk I, Drillenburg P, Peppelenbosch MP, van Deventer SJ. Dichotomal role of inhibition of p38 MAPK with SB 203580 in experimental colitis. Gut. 2002;50(4):507-12.

64. Young PR, McLaughlin MM, Kumar S, Kassis S, Doyle ML, McNulty D, et al. Pyridinyl imidazole inhibitors of p38 mitogenactivated protein kinase bind in the ATP site. J Biol Chem 1997;272(18):12116-21.

65. Lee YB, Schrader JW, Kim SU. p38 map kinase regulates TNFalpha production in human astrocytes and microglia by multiple mechanisms. Cytokine. 2000;12(7):874-80.

66. Yao L, Zhu Z, Wu J, Zhang Y, Zhang H, Sun X, et al. MicroRNA124 regulates the expression of $\mathrm{p} 62 / \mathrm{p} 38$ and promotes autophagy in the inflammatory pathogenesis of Parkinson's disease. FASEB J 2019;33(7):8648-65.

67. Tong PJ, Wu CL, Wang XF, Hu HZ, Jin HT, Li CY, et al. Development and assessment of a complete-detoxication strategy for Fuzi (lateral root of Aconitum carmichaeli) and its application in rheumatoid arthritis therapy. J Ethnopharmacol 2013;146(2):56271.

68. Shibata K, Sugawara T, Fujishita K, Shinozaki Y, Matsukawa T, Suzuki T, et al. The astrocyte-targeted therapy by Bushi for the neuropathic pain in mice. PLoS One. 2011;6(8):e23510:1-15.

69. Ito M, Komai K, Mise-Omata S, Iizuka-Koga M, Noguchi Y, Kondo T, et al. Brain regulatory T cells suppress astrogliosis and potentiate neurological recovery. Nature. 2019;565(7738):246-50.

70. Hu XM, Leak RK, Thomson AW, Yu F, Xia YG, Wechsler LR, et al. Promises and limitations of immune cell-based therapies in neurological disorders. Nat Rev Neurol 2018;14(9):559-68.

71. Hickman S, Izzy S, Sen P, Morsett L, El Khoury J. Microglia in neurodegeneration. Nat Neurosci 2018;21(10):1359-69.

72. Salter MW, Stevens B. Microglia emerge as central players in brain disease. Nat Med 2017;23(9):1018-27.

73. Prinz M, Jung S, Priller J. Microglia Biology: One Century of Evolving Concepts. Cell. 2019;179(2):292-311.

74. Li Q, Barres BA. Microglia and macrophages in brain homeostasis and disease. Nat Rev Immunol 2018;18(4):225-42.

75. Valera E, Spencer B, Masliah E. Immunotherapeutic approaches targeting amyloid-beta, alpha-synuclein, and tau for the treatment of neurodegenerative disorders. Neurotherapeutics. 2016;13(1): 179-89.

76. Singh SK, Srivastav S, Castellani RJ, Plascencia-Villa G, Perry G. Neuroprotective and antioxidant effect of Ginkgo biloba extract against $\mathrm{AD}$ and other neurological disorders. Neurotherapeutics. 2019;16(3):666-74.

Publisher's Note Springer Nature remains neutral with regard to jurisdictional claims in published maps and institutional affiliations. 